\title{
MicroRNA-374a Inhibits Aggressive Tumor Biological Behavior in Bladder Carcinoma by Suppressing Wnt/ $\beta$-Catenin Signaling
}

\author{
Xiaoliang Chen ${ }^{\mathrm{a}} \quad$ Chunshu Jia $^{\mathrm{b}}$ Chunyi Jia ${ }^{\mathrm{c}}$ Xingyi Jin ${ }^{\mathrm{d}} \quad$ Xinquan $\mathrm{Gu}^{\mathrm{a}}$ \\ aDepartment of Urology, China-Japan Union Hospital, Jilin University, Changchun, ${ }^{b}$ Center for \\ Reproductive Medicine and Center for Prenatal Diagnosis, First Hospital, Jilin University Changchun, \\ 'Department of Thoracic Oncosurgery, Jilin Province Cancer Hospital, Changchun, dDepartment of \\ Neurosurgery, China-Japan Union Hospital, Jilin University, Changchun, China
}

\section{Key Words}

Mir-374a $•$ Bladder cancer $•$ Metastasis $\cdot$ WNT5A $\bullet$ Wnt $/ \beta$-catenin

\begin{abstract}
Background/Aims: microRNA (miR)-374a plays a crucial role in cancer progression by promoting the metastasis and proliferation of various types of malignant tumors. Because its role in bladder cancer is unknown, we investigated whether miR-374a affects the progression of bladder cancer and studied the underlying mechanism. Methods: The Cancer Genome Atlas was used to analyze the clinical relevance of miR-374a. Quantitative PCR, western blotting, and luciferase and immunofluorescence assays were used to detect the expression patterns, downstream targets, and function of miR-374a in bladder cancer cells. Apoptosis was evaluated by flow cytometry after cisplatin treatment. Results: Via in silico analysis, low levels of miR-374a were associated with poor prognosis in bladder cancer patients with distant metastasis. WNT5A was a direct target of miR-374a in two bladder cancer cell lines. miR-374a mimic abrogated the metastatic potential and invasiveness of bladder cancer cells via WNT5A downregulation in both T24 and TCCSUP human bladder cancer cells; the opposite was observed with miR-374a inhibitor. In addition, miR-374a treatment reduced the phosphorylation and nuclear translocation of $\beta$-catenin. Cisplatin treatment significantly increased the apoptosis rate. Expression levels of cancer stemness-related proteins were reduced in miR-374a mimic-pretreated cells. Conclusion: Lower expression of miR-374a is associated with poor prognosis and miR-374a improves tumor biological behavior in bladder cancer cells, suggesting that miR-374a might be a novel small-molecule therapeutic target.
\end{abstract}




\section{Introduction}

Bladder cancer is the fifth most common malignant tumor in the US and is responsible for considerable health-related economic burden [1,2]. Although the percentage mortality associated with this disease remains unchanged or has even slightly decreased due to therapeutic improvements, certain patients with genetic variations or deregulated transcription show poor prognosis [3-6]. Transcriptional dysregulation results in aberrant histone methylation and microRNA (miRNA) expression [7, 8]. Previous studies have shown that PRDM2 methylation is associated with tumor recurrence [7] and that the expression levels of miRNA (miR)-337, miR-3913-2, and miR-497 can be used to calculate a risk score and predict prognosis [9-11]. Therefore, potential biomarkers related to disease prognosis and its underlying mechanism in bladder cancer should be investigated to identify novel drug targets for these patients.

Dysregulation of miRNA expression in bladder cancer results in aberrant activation of pathways involving oncogenes or inhibition of anti-tumor gene expression and signaling [12]. Dysregulation of microRNA expression may lead to alterations in the expression of genes encoding Androgen Receptor (AR) and p53 in non-muscle invasive bladder cancer (NMIBC) and muscle invasive bladder cancer (MIBC), respectively [13]. For example, miR124 , which target $A R$ in vitro, are downregulated in bladder cancer cells; this leads to aberrant expression of AR [14]. Furthermore, miR-21 upregulation in MIBC decreases p53 expression and promotes metastasis and muscular invasion. Thus, dysregulation of miRNA transcription in bladder cancer adversely influences tumor behavior [15].

miR-374a, a miRNA upregulated in breast cancer and other malignant tumors, is a tumor-promoting small non-coding RNA $[16,17]$. In breast cancer, miR-374a specifically targets WNT5, PTEN, and WIF1 genes, which are linked (by bioinformatic analysis) to negative regulation of the canonical Wnt/ $\beta$-catenin signaling pathway [16]. This activates Wnt signaling, which induces the epithelial-mesenchymal transition and eventually increases the metastatic ability of breast cancer cells. WNT5A, a noncanonical member of the Wnt ligand family, is involved in the phosphorylation of $\beta$-catenin and inhibits the canonical Wnt signaling pathway in breast cancer $[18,19]$. Unexpectedly, data mining in The Cancer Genome Atlas (TCGA) showed that miR-374a levels were low in patients with a poor prognosis. Hence, this miRNA might be a biomarker associated with better prognosis. A previous study showed that WNT5A treatment increases the migration ability of both T24 and J28 cell lines and that its expression is positively correlated with the pathological grade of tumors, indicating that WNT5A is a tumor-promoting factor in bladder cancer [20]. In addition, the anti-tumor gene PTEN is negatively correlated with reduced aggressiveness and recurrence risk in bladder cancer [21]. Nonetheless, the role of WIF1 in bladder cancer was still unknown.

Therefore, in this study, we performed a series of in vitro experiments to determine whether miR-374a inhibits the Wnt/ $\beta$-catenin pathway by targeting $W N T 5 A$ and abrogates the aggressive biological behavior and cancer stemness of bladder cancer cells. WIF1 and PTEN expression were unaltered by miR-374a treatment, whereas the miR-374a mimic suppressed WNT5A, indicating that, in contrast to previous reports, miR-374a might be a novel therapeutic target for bladder cancer.

\section{Materials and Methods}

\section{Cell culture}

Human normal urethral epithelium cell lines and various types of bladder cancer cell lines (including HTB-9, T24, and TCCSUP) were purchased from Shenjie Biotechnology (Shanghai, China). Cells were cultured in Roswell Park Memorial Institute (RPMI)-1640 medium containing 10\% fetal bovine serum (FBS) (Invitrogen, Carlsbad, CA, USA). In addition, the culture medium was supplemented with $1000 \mathrm{U} / \mathrm{L}$ each of penicillin and streptomycin and maintained at $37^{\circ} \mathrm{C}$ in a humidified (95\%) incubator containing $5 \% \mathrm{CO}_{2}$.

\section{KARGER}




\section{Cellular Physiology Cell Physiol Biochem 2018;48:815-826

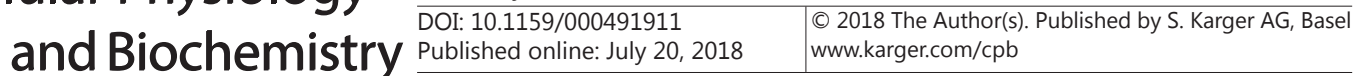 \\ Chen et al.: MiR-374a Inhibits Bladder Carcinoma}

\section{Transfection}

Invitrogen was authorized to construct the adenoviruses encoding human-WNT5A (Ad-WNT5A) and GFP (Ad-GFP, negative control). When T24 cells reached 80\% confluence, they were infected with AdWNT5A or control viruses using Lipofectamine 2000 (Invitrogen) according to the manufacturer's standard protocol, incubated for $24 \mathrm{~h}$, and then treated with the miR-374a mimic for $12 \mathrm{~h}$. The cells were subsequently collected and lysed for various assays.

\section{RNA extraction and quantitative PCR}

After treatment with the miR-374a mimic, inhibitor, or scrambled control, total RNA was isolated from human urethral epithelial cells (HUEpCs) and bladder cancer cells using TRIzol reagent (Invitrogen) according to the manufacturer's instructions. The total RNA was reverse transcribed using hexamer primers and Superscript III reverse transcriptase (Invitrogen) for analysis of transcript levels. Subsequently, quantitative polymerase chain reaction (qPCR) was performed using the SYBR Green PCR Master Mix. miRNA transcriptional analysis was performed after reverse transcription, followed by qPCR, as described before [22]. qPCR for miRNAs was performed with the miScript SYBR Green PCR kit (Qiagen, Shanghai, China). GAPDH mRNA and U6 small RNA were used as endogenous controls for mRNA and miRNA expression, respectively. The relative expression level was calculated using the $2^{-\Delta \Delta \mathrm{Ct}}$ method. The sequences of the upstream and downstream primers were as follows: WNT5A: F-5'ACACGATGGT CAAAGCTAGT CGTAG-3', R-5'ACGGTCAGTT GTTCAGTTCC-3'; GAPDH: F-5'CAAGGTCATC CATGACAACTT TG-3', R-5'GTCCACCACC CTGTTGCTGT AG-3'; U6: F-5'CTCGCTTCGG CAGCACA-3', R-5'AACGCTTCAC GAATTTGCGT-3'.

\section{Western blotting}

Western blotting was performed using the following primary antibodies: WNT5A (ab72583, 1:2000), $\beta$-catenin (ab32572, 1:10000), phosphorylated $\beta$-catenin (ab27798, 1:1000), ABCG2 (ab24115, 1:100), CD133 (ab19898, 1:1000), and SOX2 (ab92494, 1:2000). All primary antibodies were purchased from Abcam Plc. (Cambridge, UK). Cells were lysed with lysis buffer for $10 \mathrm{~min}$ and then centrifuged at 12, $000 \times \mathrm{g}$ for $10 \mathrm{~min}$ at $4^{\circ} \mathrm{C}$. The concentration of the protein lysates was estimated using BioTek EPOCH (Winooski, VT, USA). Protein samples were heat-denatured at $100^{\circ} \mathrm{C}$ for $5 \mathrm{~min}$. Equivalent protein lysates were separated by $10 \%$ sodium dodecyl sulfate-polyacrylamide gel electrophoresis and then transferred to nitrocellulose membranes. The membranes were first blocked with $5 \%(\mathrm{w} / \mathrm{v})$ dried skim milk in Tris-buffered saline with Tween 20 (TBST) for $1 \mathrm{~h}$ at $25^{\circ} \mathrm{C}$ and then probed with the indicated primary antibodies overnight at $4^{\circ} \mathrm{C}$. Next, the membranes were washed thrice with $1 \times$ TBST and incubated with the corresponding horseradish peroxidase-conjugated secondary antibodies for $2 \mathrm{~h}$ at room temperature. $\beta$-actin (anti- $\beta$-actin from Kangcheng, Inc., Shanghai, China) was used as an internal control. After three washes with TBST, the proteins were detected using an enhanced chemiluminescence system (Pierce Biotechnology, Rockford, IL, USA) and Alpha Innotech Fluorchem SP (San Leandro, CA, USA).

\section{Wound-healing assay}

A wound-healing assay was performed to evaluate cell migration. Cells were seeded on 6-well plates in RPMI-1640 medium containing 10\% FBS. After $24 \mathrm{~h}$, straight lines were drawn by scraping the confluent cells with a $20-\mu \mathrm{L}$ pipette tip and ruler. Subsequently, the medium and floating cells were carefully removed and the adherent cells were washed thrice with PBS and cultured in serum-free medium. After $16 \mathrm{~h}$ of incubation, the wound-healing process was monitored under a phase contrast microscope. The migration distance was measured using representative images obtained at 0 and $16 \mathrm{~h}$.

\section{Cell migration and invasion assays}

Transwell assays were performed to assess cell invasion using the HTS Transwell-24 system (Corning Inc., Corning, NY, USA). Cells were cultured in serum-free RPMI-1640 medium for $24 \mathrm{~h}$. Then, $100 \mu \mathrm{L}$ of $5 \times 10^{4}$ cells were seeded onto the upper chamber, and the lower chamber was filled with $600 \mu \mathrm{L}$ of RPMI1640 medium supplemented with $10 \%$ FBS as a chemoattractant. The cells in the upper chamber (without serum) have a tendency to pass through the membrane to the culture medium containing $10 \%$ FBS in the lower compartment, allowing an estimation of migratory and invasive abilities. After incubation at $37^{\circ} \mathrm{C}$ for $24 \mathrm{~h}$, the cells that passed through the membrane and were in the lower compartment were stained with $0.2 \%$ crystal violet, fixed with $30 \%$ glycerin on the slides, and then counted under a light microscope. 


\section{Cellular Physiology Cell Physiol Biochem 2018;48:815-826 \begin{tabular}{l|l} 
DOI: 10.1159/000491911 & and Biochemistry \\
Published online: July 20, 2018 & $\begin{array}{l}\text { 2018 The Author(s). Published by S. Karger AG, Basel } \\
\text { www.karger.com/cpb }\end{array}$
\end{tabular} \\ Chen et al.: MiR-374a Inhibits Bladder Carcinoma}

\section{Immunofluorescence}

Cells were adhered on coverslips in 6-well plates, and after $24 \mathrm{~h}$ in culture, were fixed with $4 \%$ paraformaldehyde for $15 \mathrm{~min}$, permeabilized with $0.2 \%$ Triton X-100 for $15 \mathrm{~min}$, and blocked with phosphate buffer containing 3\% bovine serum albumin for $30 \mathrm{~min}$ at room temperature. After incubation with rabbit anti- $\beta$-catenin (Abcam) overnight at $4^{\circ} \mathrm{C}$, the coverslips were incubated with CY3-conjugated goat antirabbit IgG secondary antibodies for $30 \mathrm{~min}$ and treated with 4',6-diamidino-2-phenylindole (DAPI) (SigmaAldrich, St. Louis, MO, USA) for nuclear staining. The cells were examined using confocal laser scanning microscopy (Leica, Wetzlar, Germany).

\section{Cell viability assay and chemotherapeutic drug treatment}

Cell viability was evaluated using flow cytometry according to standard protocols. In total, $10^{4}$ cells were treated with the miR-374a mimic or control oligonucleotides and seeded in 96-well plates, and CDDP ( $1 \mu \mathrm{mol} / \mathrm{L}$, Sigma) was added for 2-4 days. Cells were harvested and washed with PBS $48 \mathrm{~h}$ after transfection. Cells $\left(\sim 0.5 \times 10^{6}\right)$ were resuspended in the flow cytometry staining buffer (eBioscience, Frankfurt, Germany) and stained with annexin V-FITC/propidium iodide (PI) (eBioscience) in the dark for $30 \mathrm{~min}$. Cell apoptosis was detected by flow cytometry according to the annexin V-FITC/PI kit protocol and detected using CellQuest Pro software (BD Biosciences, San Jose, CA, US). The flow cytometry data were analyzed using FlowJo 6.0 (BD Biosciences).

\section{TCGA database}

We used the public website PROmiR2 (http://xvm145.jefferson.edu/progmir/index.php) to determine whether miR-374a exerted any positive influence on the prognosis of patients with bladder cancer. The raw data are freely available on the website of TCGA database. The website directly offers the survival curve and statistical results according to the survival data. We downloaded the survival data and analyzed the corresponding figures using the Kaplan-Meier method, followed by a log-rank test.

\section{Statistical analysis}

Each experiment was repeated at least three times and the data are presented as the mean \pm standard error of the mean (SEM). Unpaired t-tests were used to determine the statistical differences between two groups in each analysis. $\mathrm{P}<0.05$ was considered statistically significant. All statistical analyses were performed using SPSS 13.0 software (SPSS Inc., Chicago, IL, USA).

\section{Results}

\section{Analysis of TCGA database}

We performed survival analysis using TCGA database to determine whether miR-374 can be used to predict clinical prognosis. First, we categorized patients with bladder cancer into two groups according to miR-374a level (high or low) based on the recommended standard on the PROGmiR2 website (http://xvm145.jefferson.edu/progmir/index.php) [23]. Next, we analyzed the survival data in different subgroups based on the extent of metastasis. We observed that poor overall survival (OS) in patients with lower levels of miR-374a in the subgroup with metastasis (odds ratio $[\mathrm{OR}]=0.6,95 \%$ confidence interval [CI]: 0.39-0.93, $P=0.02$ ), and a difference was observed in relapse-free survival (RFS) between the two subgroups $(\mathrm{P}=0.68)$ (Fig. $1 \mathrm{~A}$ and $1 \mathrm{~B})$. In the non-metastasis subgroup, poor RFS was seen in the subgroup with lower levels of miR-374a (OR $=0.35,95 \% \mathrm{CI}: 0.21-0.6, \mathrm{P}<0.01)$, although the OR of the two subgroups was similar (Fig. 1C and 1D). In the combined patient group, neither OS nor RFS differed between the subgroups (Fig. 1E and 1F), which demonstrated that metastasis should be considered in survival analysis because of the poor prognosis of patients with distant metastasis.

miR-374a directly targets WNT5A bladder cancer cells

We measured the miR-374a transcript levels in normal HUEpCs and bladder cancer cells by qPCR to determine whether miR-374a transcription was reduced in cancer cells. 


\section{Cellular Physiology Cell Physiol Biochem 2018;48:815-826 \begin{tabular}{l|l} 
DOI: 10.1159/000491911 & $\begin{array}{l}\text { O 2018 The Author(s). Published by S. Karger AG, Basel } \\
\text { www.karger.com/cpb }\end{array}$
\end{tabular} \\ Chen et al.: MiR-374a Inhibits Bladder Carcinoma}

Fig. 1. Transcript levels of miR-374a positively influence the prognosis of metastatic patients with bladder cancer. (A) Transcript levels of miR-374a positively influence overall survival in metastatic patients with bladder cancer according to survival analysis. (B) Transcript levels of miR-374a positively influence RFS in metastatic patients with bladder cancer according to survival analysis. (C) Transcript levels of miR-374a have no influence on overall survival in non-metastatic patients with bladder cancer according to survival analysis. (D) Transcript levels of miR-374a positively influence RFS in non-metastatic patients with bladder cancer according to survival analysis. (E) Transcript levels of miR-374a have no influence on overall survival in combined patients with bladder cancer according to survival analysis. (F) Transcript levels of miR-374a have no influence on RFS in combined patients with bladder cancer according to survival analysis. miR-374a expression was based on the median values in the population. The red line indicates the high expression subgroup and the green line indicates the low expression subgroup.
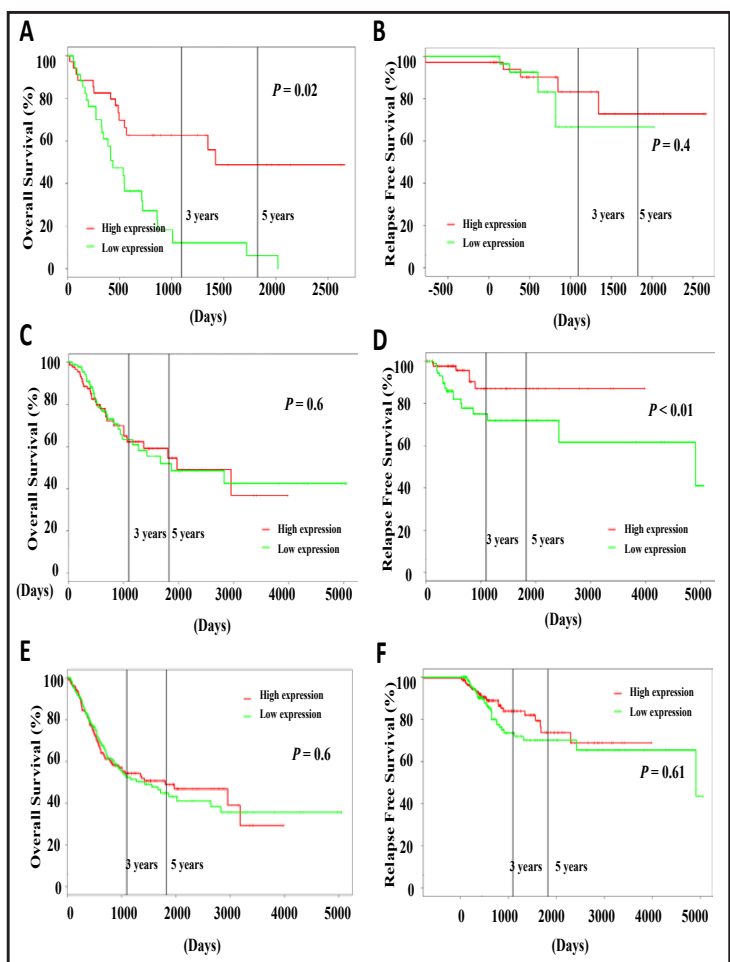

Unexpectedly, the miR-374a transcript level was similar in HTB-9 cells and HUEpCs, but the transcript levels were significantly lower in T24 and TCCSUP cells than in HUEpCs (Fig. 2A). These results indicate the heterogeneity of miR-374a transcription among various bladder cancer cell lines, which is reminiscent of the differential transcription levels observed in clinical samples. Additionally, we observed high metastatic activity in T24 cells compared with HTB-9 cells (data not shown). Therefore, we selected the T24 and TCCSUP cells to further investigate the role of miR-374a in bladder cancer.

We used western blot to detect the expression of the potential targets of miR-374a, such as PTEN, WIF1, and WNT5A. PTEN expression was low in T24 cells but not in TCCSUP cells, whereas WNT5A was highly expressed in both cell lines (Fig. 2B). We hypothesized that miR374 a did not influence PTEN and WIF1 expression in bladder cancer cells. To further validate this theory, we performed qPCR in T24 and TCCSUP cells after treatment with a miR-374a mimic. As expected, the miR-374a mimic decreased the expression of WNT5A alone and not PTEN and WIF1 (Fig. 2C and 2D). Thus, unlike in breast cancer cells, the miR-374a mimic targeted WNT5A for downregulation in bladder cancer cells, without affecting PTEN and WIF1 expression. Furthermore, a dual-luciferase reporter system with vectors comprising the mutant or wild-type WNT5A 3'-UTR were used to confirm if WNT5A is a direct target of miR-374a (Fig. 2E). We observed decreased relative wild-type WNT5A 3'-UTR luciferase activities in both T24 and TCCSUP cells lines. However, the mutant luciferase activities were unchanged after miR-374a mimic treatment (Fig. $2 \mathrm{~F}$ and $2 \mathrm{G}$ ). These results suggested that WNT5A is a direct target of miR-374a.

miR-374a suppresses the metastatic potential and invasiveness of bladder cancer cells via WNT5A downregulation

As shown in Fig. 3A, WNT5A transcript was greatly upregulated after introduction of an adenovirus vector overexpressing WNT5A into T24 cells. Functionally, overexpression of WNT5A aggravated the metastasis and invasiveness of T24 cells in wound-healing and Transwell assays (Fig. 3B-D). In addition, treatment with the miR-374a mimic significantly abrogated the metastatic ability and invasiveness of T24 cells (Fig. 3B-D). Thus, both miR374 and WNT5A can alter bladder cell biology. 


\section{Cellular Physiology Cell Physiol Biochem 2018;48:815-826 \begin{tabular}{l|l} 
DOI: 10.1159/000491911 & O 2018 The Author(s). Published by S. Karger AG, Basel \\
www.karger.com/cpb
\end{tabular}

Fig. 2. miR-374a directly targets WNT5A in bladder cancer cells. (A) The expression level of miR-374a was measured in HUEpCs, HTB9, TCCSUP, and T24 cells using qPCR. (B) Protein levels of the indicated genes in HUEpCs, TCCSUP, and T24 cells were measured by western blot. (C) mRNA levels of the indicated genes treated with or without $50 \mathrm{nM}$ miR374 a mimic in T24 cells were measured by qPCR. (D) mRNA levels of the indicated genes treated with or without $50 \mathrm{nM}$ miR-374a mimic in TCCSUP cells were measured by qPCR. (E) Mutant (Mut) and wild-type (WT) WNT5A 3'-UTR were compared. (F) Luciferase activity was detected in T24 cells transfected with reporter vectors consisting of the wildtype 3'-UTR (or mutant 3'-UTR) of WNT5A. (G) Luciferase activity was measured in TCCSUP cells transfected with reporter vectors consisting of the wild-type 3'-UTR (or mutant 3'-UTR) of WNT5A. The results from three independent experiments are described as the mean \pm standard error. ${ }^{*} \mathrm{P}<0.05,{ }^{* *} \mathrm{P}<0.01$, and $* * * \mathrm{P}<0.001$.

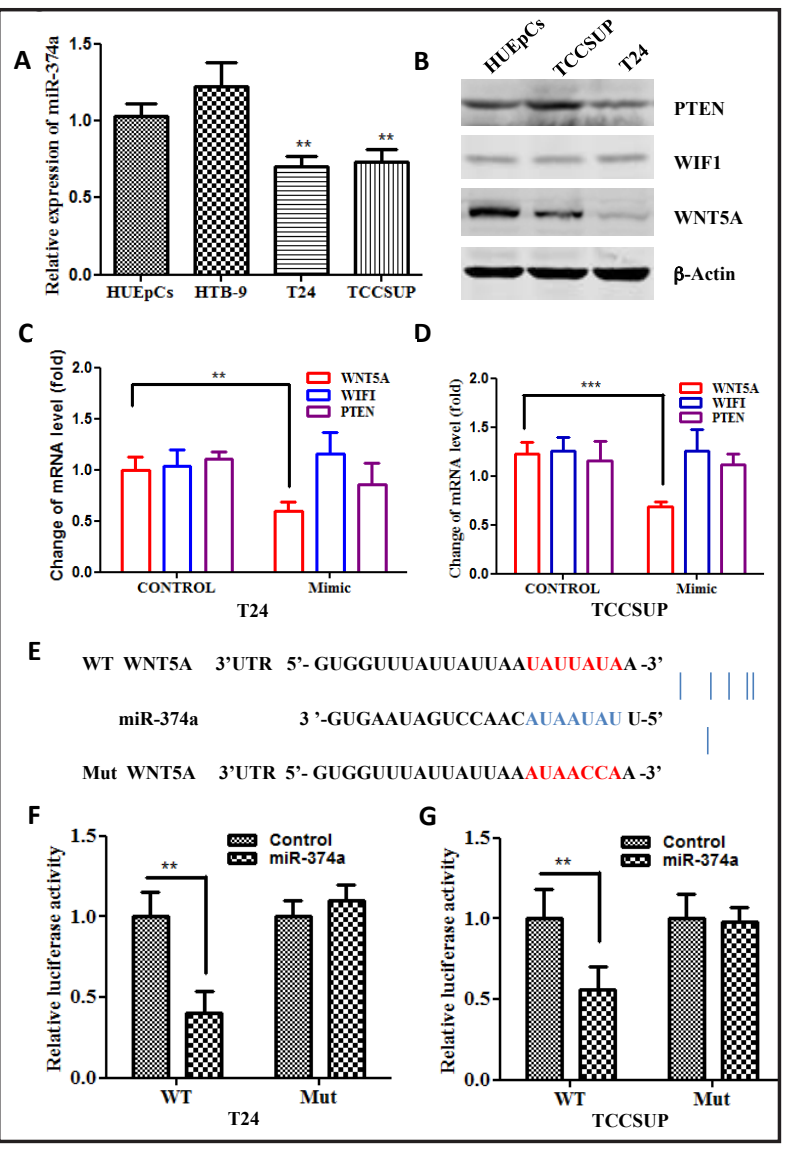

To determine whether the effect of miR-374a on cancer cell biology was via WNT5A downregulation, T24 cells overexpressing WNT5A were treated with miR-374a mimic. First, we observed that miR-374a mimic significantly abrogated the increase in WNT5A (Fig. 3B-D). Second, the increased metastasis and invasiveness of T24 cells induced by WNT5A overexpression were also suppressed (Fig. 3B-D). Similar results were observed in TCCSUP cells (Fig. 3E-H).

In addition, treatment of T24 and TCCSUP cells with miR-374a inhibitor significantly increased the metastatic ability and invasiveness of T24 cells, which were accompanied by increased WNT5A transcript (Fig. 4). Taken together, these data suggested that miR-374a inhibited metastasis and invasiveness in vitro via WNT5A downregulation.

miR-374a-induced downregulation of WNT5A inhibits the canonical Wnt/ $\beta$-catenin signaling pathway

We further investigated whether regulation of miR-374a and WNT5A levels was associated with Wht noncanonical signaling. Previous work showed that WNT5A, a noncanonical ligand of the Wnt family, plays opposing roles in cancer biology [24]. However, one recent study showed that WNT5A activates phosphokinase A to deactivate GSK3 $\beta$ and stabilize $\beta$-catenin, which in turn activates the canonical Wnt signaling pathway [25]. We measured the phosphorylation and protein levels of $\beta$-catenin after introduction of the WNT5A-overexpressing adenovirus vector. The results revealed decreased phosphorylation but similar protein levels of $\beta$-catenin, which showed that WNT5A treatment did not influence $\beta$-catenin transcription (Fig. 5A). Moreover, phosphorylation of GSK3 $\beta$ was increased, which suggested GSK3 $\beta$ inactivation [26].

Next, we performed western blotting and immunofluorescence assays to measure the activation of the Wnt signaling pathway after introduction of miR-374a. We observed 
Fig. 3. miR-374a mimic decreases metastatic and invasive abilities in bladder cancer cells via degeneration of WNT5A. (A) Adenovirus vector encoding WNT5A was infected into T24 cells for $24 \mathrm{~h}$; the cells were then treated with or without $50 \mathrm{nM}$ miR-374a mimic. mRNA levels of WNT5A were measured using distance in 16-h wound-healing assays under the same experimental conditions as (A). (C) Quantified data of Transwell assays under the same experimental conditions as (A). (D) Representative images of Transwell assays under the same experimental conditions as (A). (E) Adenovirus vector encoding WNT5A was infected into TCCSUP cells for $24 \mathrm{~h}$; the cells were then treated with or without $50 \mathrm{nM}$ miR-374a mimic. mRNA levels of WNT5A were measured using qPCR. (F) Quantified data of migration distance in 16-h wound-healing assays under the same experimental conditions as (E). (G) Quantified data of Transwell assays under the same experimental conditions as (E). (H) Representative images of Transwell assays under the same experimental conditions as (E). The results from three independent experiments are described ${ }^{* *} \mathrm{P}<0.01$, and ${ }^{* * *} \mathrm{P}<0.001$. qPCR. (B) Quantified data of migration as the mean \pm standard error. ${ }^{*} \mathrm{P}<0.05$,
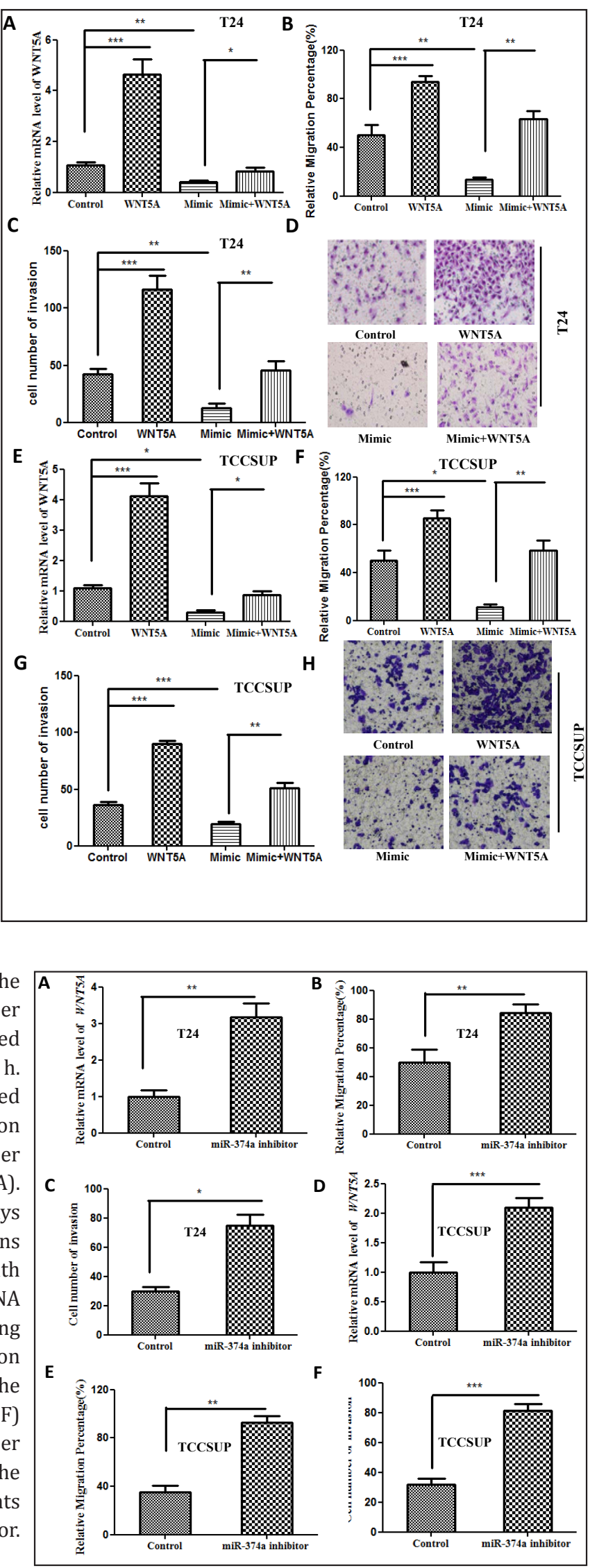

Fig. 4. miR-374a inhibitor increases the metastatic and invasive abilities of bladder cancer cells. (A) T24 cells were treated with $100 \mathrm{nM}$ miR-374a inhibitor for $24 \mathrm{~h}$. mRNA levels of WNT5A were measured using qPCR. (B) Quantified data of migration distance in wound-healing assays under the same experimental conditions as (A). (C) Quantified data of Transwell assays under the same experimental conditions as (A). (D) TCCSUP cells were treated with $100 \mathrm{nM}$ miR-374a inhibitor for $24 \mathrm{~h}$. mRNA levels of WNT5A were measured using qPCR. (E) Quantified data of migration distance in wound-healing assays under the same experimental conditions as (D). (F) Quantified data of Transwell assays under the same experimental conditions as (E). The results from three independent experiments are described as the mean \pm standard error. ${ }^{*} \mathrm{P}<0.05,{ }^{* *} \mathrm{P}<0.01$, and ${ }^{* * *} \mathrm{P}<0.001$.
KARGER 


\section{Cellular Physiology Cell Physiol Biochem 2018;48:815-826 \begin{tabular}{l|l} 
DOI: 10.1159/000491911 & $\begin{array}{l}\text { O } 2018 \text { The Author(s). Published by S. Karger AG, Basel } \\
\text { www.karger.com/cpb }\end{array}$
\end{tabular} Chen et al.: MiR-374a Inhibits Bladder Carcinoma}

increased nuclear translocation and reduced phosphorylation of $\beta$-catenin after introduction of the miR-374a mimic into T24 cells (Fig. 5B and 5C). Introduction of miR-374a into T24 cells increased the phosphorylation levels of $\beta$-catenin and reduced its nuclear translocation, which suggested that miR374 a could inhibit the canonical Wnt signaling pathway (Fig. 5C). Moreover, overexpression of WNT5A reversed the inhibition of $W n t / \beta$-catenin signaling observed after treatment with the miR$374 \mathrm{a}$ mimic, which demonstrated that WNT5A activated the canonical Wnt/ $\beta$ catenin signaling pathway in bladder cancer cells (Fig. 5B and 5C). Overall, our data showed that the noncanonical ligand WNT5A activated the tumor-promoting canonical Wnt signaling pathway in the same way as observed in human dermal fibroblasts [27].

Then, we used CP21, a highly selective inhibitor of GSK3 $\beta$, to activate Wht signaling and investigate its effect on the metastatic and invasive abilities of the T24 cell line after treatment with the miR-374a mimic. CP21 partially restored metastatic ability and invasiveness on Transwell and wound-healing assays after treatment with miR-374a (Fig. 5D and $5 \mathrm{E}$ ). These results indicated that miR374a-induced downregulation of WNT5A inhibited the canonical Wnt/ $\beta$-catenin signaling pathway.

miR-374a mimic reduces cancer stemness and increases sensitivity to cisplatin

We investigated whether the miR374a mimic could alter cancer stemness and sensitivity to chemotherapeutic drugs in bladder cancer cell lines via

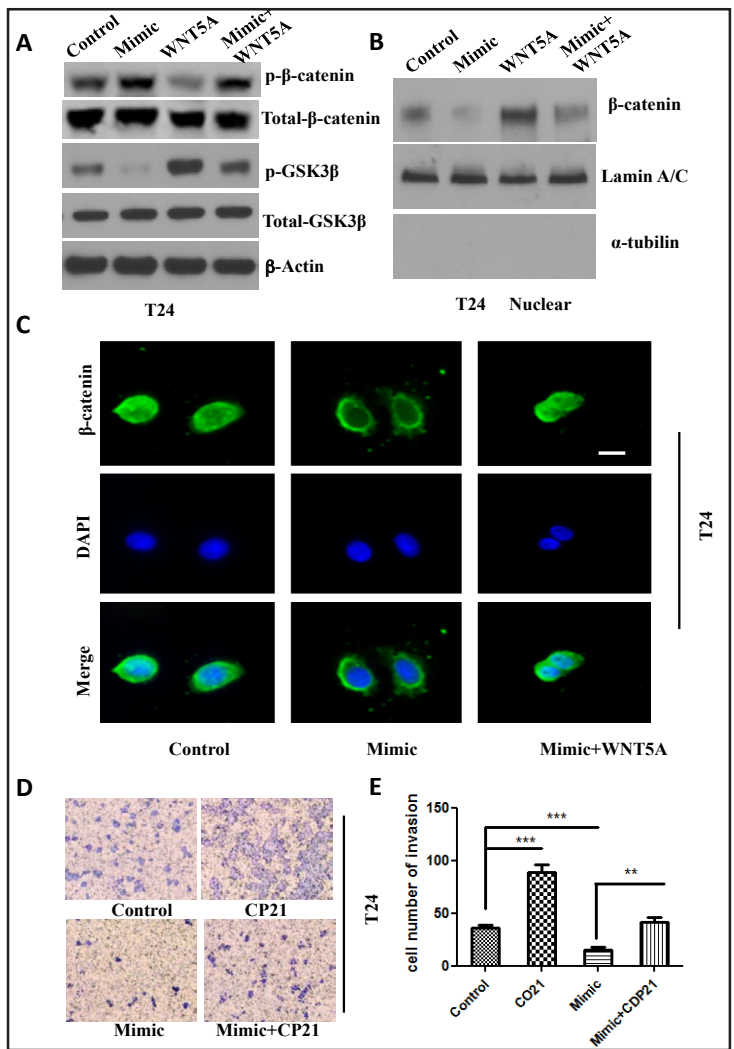

Fig. 5. miR-374a mimic suppresses the canonical Wnt/ $\beta$-catenin signaling pathway by targeting WNT5A. (A) Adenovirus vector encoding WNT5A was infected into T24 cells for $24 \mathrm{~h}$; the cells were then treated with or without $50 \mathrm{nM}$ miR-374a mimic. Protein levels of the indicated genes in T24 cells were measured by western blot. (B) Protein levels of $\beta$-catenin in the nuclear fraction of T24 cells were measured by western blot. (C) Immunofluorescence staining of T24 cells under the same experimental conditions as (A) with $\beta$-catenin (green) and DAPI (blue). Bar, $100 \mu \mathrm{m}$. (D) T24 cells were treated with $50 \mathrm{nM}$ miR-374a mimic, $1 \mu \mathrm{M}$ CP21, or their combination for $24 \mathrm{~h}$. Representative images of Transwell assays are shown. (E) Quantified data of the Transwell assays shown in (D). The results from three independent experiments are described as the mean \pm standard error. ${ }^{* *} \mathrm{P}<0.01$ and ${ }^{* * *} \mathrm{P}<0.001$. inhibition of the Wnt signaling pathway. Introduction of the miR-374a mimic blocked the Wnt signaling pathway, which has multiple effects on tumor biology, including metastasis, cell proliferation, cancer cell stemness, and neovascularization in the carcinoma microenvironment $[28,29]$. We speculated that the miR-374a treatment negatively regulated not only metastasis and invasion, but also other biological mechanisms associated with poor prognosis because we observed poor prognosis in patients with lower miR-374a levels in the distant metastasis subgroups from the survival data. Hence, we considered increased chemotherapeutic sensitivity to be another possible phenotypic conversion mechanism after blockade of Wnt signaling in bladder cancer cell lines. We measured the apoptosis rate and sensitivity to cisplatin by flow cytometry and found that pretreatment with the miR-374a mimic significantly promoted apoptosis of bladder 


\section{Cellular Physiology Cell Physiol Biochem 2018;48:815-826 \begin{tabular}{c|c} 
DOI: 10.1159/000491911 & $\begin{array}{l}\text { O 2018 The Author(s). Published by S. Karger AG, Basel } \\
\text { www.karger.com/cpb }\end{array}$
\end{tabular}

cancer cells compared with the control group (Fig. 6A and 6B). Furthermore, we observed reduced expression of gene markers related to cancer cell stemness, including SOX2, $C D 133$, and $A B C G 2$, indicating that miR-374a deficiency might be involved in the chemotherapeutic resistance via enhancement of cancer cell stemness (Fig. 6C and 6D). These observations illustrated that the miR-374a mimic could block the Wnt/ $\beta$-catenin signaling pathway to regulate different tumor behaviors, including metastasis, invasion, cancer stemness, apoptosis, and drug resistance.

\section{Discussion}
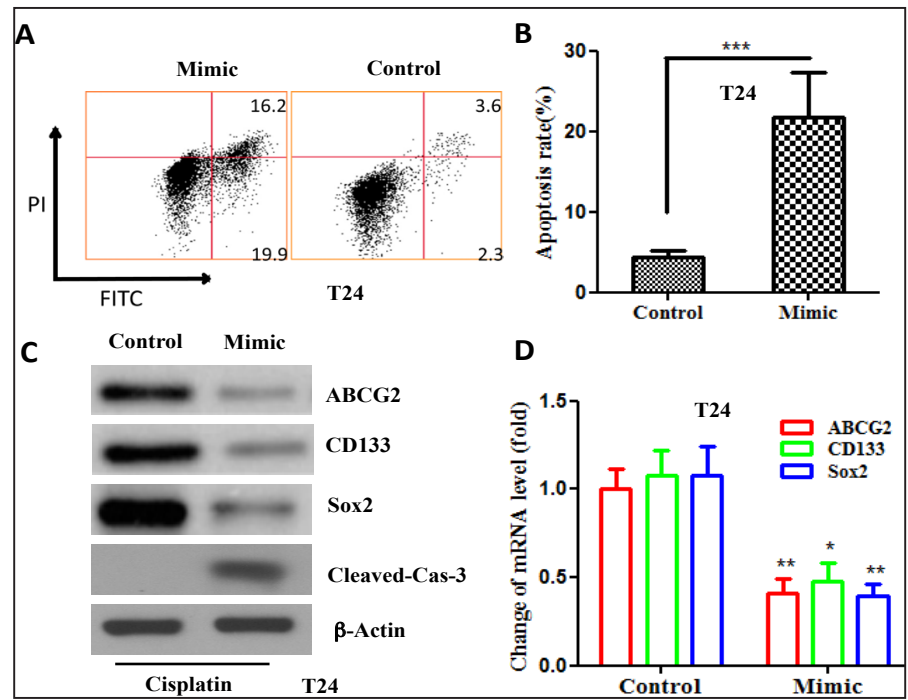

Fig. 6. miR-374a mimic reduces cancer stemness and enhances sensitivity to cisplatin. (A) Representative Fig. of annexin V/PI in flow cytometry analysis and the data indicating an increased apoptosis rate induced by $5 \mu \mathrm{M}$ cisplatin after treatment with or without $50 \mathrm{nM}$ miR-374a mimic. (B) Quantified data of (A). (C) Protein levels of the indicated genes in T24 cells under the same experimental conditions as (A) were measured by western blot.

Although numerous (D) mRNA levels of the indicated genes in T24 cells under the same experimental conditions as (A) were measured by qPCR. The results from three independent experiments are described as the mean \pm standard error. ${ }^{*} \mathrm{P}<0.05,{ }^{* *} \mathrm{P}<0.01$, and ${ }^{* * *} \mathrm{P}<0.001$.

the

deregulation in bladder cancer,

including the overexpression

or deficiency of miRNAs and

abnormal methylation levels of target genes, further in-depth investigation of the mechanisms underlying abnormal transcriptional regulation was warranted by the identification of personalized transcriptional deregulation in various types of cancer and the complicated functions of miRNAs $[8,16]$. Dysregulation of miRNA expression varies among different types of cancer. Yoshino et al [30]. found that miR-99, miR-100, miR-101, and miR-145 were downregulated in NMIBC but not in MIBC using miRNA microarray. miRNA dysfunction is also dependent on the pathological grade of bladder cancer. High-grade bladder carcinomas (MIBC or NMIBC) share an aberrant miRNA transcription profile, which is distinct from that of their low-grade counterparts. This similarity in the miRNA profile assists clinicians in determining the therapy required for patients with high-grade NMIBC. However, because abnormal gene expression varies with the pathological grade and clinical type of tumor, the intervention strategy for tumors with different biological features would also vary.

In this study, we observed that the transcript levels of miR-374a were positively correlated with disease prognosis. Therefore, we hypothesized that miR-374a might act as an anti-tumor small non-coding RNA. After adjustment for metastatic status, patients with high levels of miR-374a have longer survival and RFS times. NMIBC patients with high levels of miR-374a have a longer RFS, which suggests that miR-374a inhibits tumor metastasis and aggressive biological behavior. A previous study showed that miR-374a promoted the epithelial-mesenchymal transition in breast cancer via activation of the canonical Wnt signaling pathway, which aggravates metastasis and invasion in vitro [16]. These observations contrast with our results on the role of miR-374 in bladder cancer and its effect on disease prognosis. Previous study demonstrated that WNT5A, a noncanonical Wnt ligand, plays a vital tumor-promoting role in bladder cancer [20]. Other studies of the role of WNT5A in tumor malignancy reported inconsistent results. For example, Topol et al [31]. showed that 


\section{Cellular Physiology Cell Physiol Biochem 2018;48:815-826 \begin{tabular}{l|l} 
DOI: 10.1159/000491911 & Ond Biochemistry 2018 The Author(s). Published by S. Karger AG, Basel \\
www.karger.com/cpb
\end{tabular} \\ Chen et al.: MiR-374a Inhibits Bladder Carcinoma}

WNT5A antagonizes the canonical Wnt signaling pathway and eventually results in $\beta$-catenin downregulation. In contrast, Gujral et al [32]. found that WNT5A plays a tumor-promoting role in various cancers via an unrecognized noncanonical Wnt pathway. Binding of WNT5A to the Fzd2 receptor activates STAT3 and promotes the Janus kinase-independent epithelialmesenchymal transition. In contrast, Mikels and Nusse [33] observed that WNT5A also activated $\beta$-catenin transcription via its specific receptor (overexpressed Fz-4 and LRP5), but did not affect $\beta$-catenin phosphorylation and stability.

Nonetheless, recent studies have demonstrated that WNT5 can activate PKA and in turn inactivate GSK3 $\beta$, which leads to stabilization and enhanced nuclear translocation of $\beta$-catenin [25]. Additionally, they showed that the noncanonical Wnt ligand WNT5A can activate the canonical Wnt signaling pathway via inactivation of GSK3 $\beta$ in human dermal fibroblasts. Our results are consistent with the findings of the above study. We found that WNT5A aggravates bladder cancer via activation of the canonical Wnt signaling pathway. After considering the varying results obtained with other malignant tumors, we hypothesized that the distinct biological effects observed in different cell lines could be attributed to differences in the isoforms of the WNT5A receptors belonging to the Frizzled receptor family. In other words, the signaling pathway activated after WNT5A treatment depends on the isoform of the Frizzled receptor present in the cell type. This hypothesis might explain the contradictory results observed with WNT5A signaling in breast and bladder cancer cells.

Reports show that WNT5A has contradictory effects on tumorigenicity $[16,18,20]$. We observed that WNT5A treatment aggravated bladder cancer metastasis and invasiveness with increased phosphorylation of $\beta$-catenin. The role of WNT5A in bladder cancer is still ambiguous because of the opposing results reported in previous studies. Some groups found that WNT5A levels were positively correlated with the histological grade and clinical stage of bladder cancer and that the WNT5A mRNA levels varied widely among different cell lines [25]. Furthermore, aberrant expression of $C D C 42 B P B, W N T 5 A$, and ROBO1 could be used for the molecular diagnosis of bladder cancer [34]. Our study demonstrated that WNT5A may act as a potential biomarker and tumor-promoting factor in bladder cancer. We found that WNT5A activated the canonical Wnt signaling pathway and promoted cell invasion and metastasis, which worsened the tumor phenotype. The activation of the canonical Wnt signaling pathway also resulted in other phenotypic changes, including apoptosis, chemotherapy resistance, and cancer stemness [28]. Therefore, we performed flow cytometry to assess the apoptosis rate after treatment with cisplatin and evaluated the levels of proteins related to cancer stemness. We observed that inactivation of the canonical Wnt signaling pathway via WNT5A downregulation not only attenuated metastasis and invasion, but also reduced cancer stemness and enhanced sensitivity to cisplatin. Overall, these results demonstrated that miR-374a exerts anti-tumor effects via downregulation of WNT5A and blockade of the canonical Wnt signaling pathway in bladder cancer.

In conclusion, miR-374a exerts an anti-tumor effect in bladder cancer. miR-374a specifically targets $W N T 5 A$, a noncanonical Wnt ligand that activates canonical Wnt signaling in the presence of specific receptors in bladder cancer cells, abrogates the invasiveness and metastasis potential, and increases apoptosis after cisplatin treatment. Therefore, miR-374a may be a novel therapeutic target in bladder cancer patients with low levels of miR-374a transcripts.

\section{Disclosure Statement}

The authors have no conflicts of interest.

\section{References}




\section{Cellular Physiology Cell Physiol Biochem 2018;48:815-826 \begin{tabular}{l|l} 
DOI: 10.1159/000491911 & Ond Biochemistry \\
Published online: July 20, 2018 & $\begin{array}{l}\text { 2018 The Author(s). Published by S. Karger AG, Basel } \\
\text { www.karger.com/cpb }\end{array}$
\end{tabular} \\ Chen et al.: MiR-374a Inhibits Bladder Carcinoma}

-2 Li HJ, Sun XM, Li ZK, Yin QW, Pang H, Pan JJ, Li X, Chen W: Lncrna uca1 promotes mitochondrial function of bladder cancer via the mir-195/arl2 signaling pathway. Cell Physiol Biochem 2017;43:2548-2561.

-3 Correction: Atf3 suppresses metastasis of bladder cancer by regulating gelsolin-mediated remodeling of the actin cytoskeleton. Cancer Res 2016;76:4592.

4 Li C, Du Y, Yang Z, He L, Wang Y, Hao L, Ding M, Yan R, Wang J, Fan Z: Galnt1-mediated glycosylation and activation of sonic hedgehog signaling maintains the self-renewal and tumor-initiating capacity of bladder cancer stem cells. Cancer Res 2016;76:1273-1283.

5 Wang M, Li Z, Chu H, Lv Q, Ye D, Ding Q, Xu C, Guo J, Du M, Chen J, Song Z, Yin C, Qin C, Gu C, Zhu Y, Xia G, Liu F, Zhang Z, Yuan L, Fu G, Hu Z, Tong N, Shen J, Liu K, Sun J, Sun Y, Li J, Li X, Shen H, Xu J, Shi Y, Zhang Z: Genome-wide association study of bladder cancer in a chinese cohort reveals a new susceptibility locus at 5q12.3. Cancer Res 2016;76:3277-3284.

6 Zhi Y, Pan J, Shen W, He P, Zheng J, Zhou X, Lu G, Chen Z, Zhou Z: Ginkgolide b inhibits human bladder cancer cell migration and invasion through microrna-223-3p. Cell Physiol Biochem 2016;39:1787-1794.

7 Garcia-Baquero R, Puerta P, Beltran M, Alvarez M, Sacristan R, Alvarez-Ossorio JL, Sanchez-Carbayo M: Methylation of a novel panel of tumor suppressor genes in urine moves forward noninvasive diagnosis and prognosis of bladder cancer: A 2-center prospective study. J Urol 2013;190:723-730.

8 Nickerson ML, Dancik GM, Im KM, Edwards MG, Turan S, Brown J, Ruiz-Rodriguez C, Owens C, Costello JC, Guo G, Tsang SX, Li Y, Zhou Q Cai Z, Moore LE, Lucia MS, Dean M, Theodorescu D: Concurrent alterations in tert, kdm6a, and the brca pathway in bladder cancer. Clin Cancer Res 2014;20:4935-4948.

-9 Peng F LH, Xiao H, Li L, Li Y, Wu Y.: Identification of a three mirna signature as a novel potential prognostic biomarker in patients with bladder cancer. Oncotarget 2017;8:105553-105560.

10 Shang A, Yang M, Shen F, Wang J, Wei J, Wang W, Lu W, Wang C, Wang C: Mir-1-3p suppresses the proliferation, invasion and migration of bladder cancer cells by up-regulating sfrp1 expression. Cell Physiol Biochem 2017;41:1179-1188.

11 Li P, Yang X, Cheng Y, Zhang X, Yang C, Deng X, Li P, Tao J, Yang H, Wei J, Tang J, Yuan W, Lu Q, Xu X, Gu M: Microrna-218 increases the sensitivity of bladder cancer to cisplatin by targeting glut1. Cell Physiol Biochem 2017;41:921-932.

12 Hayes J, Peruzzi PP, Lawler S: Micrornas in cancer: Biomarkers, functions and therapy. Trends Mol Med 2014;20:460-469.

13 Fendler A, Stephan C, Yousef GM, Jung K: Micrornas as regulators of signal transduction in urological tumors. Clin Chem 2011;57:954-968.

14 Xiong Y, Wang L, Li Y, Chen M, He W, Qi L: The long non-coding rna xist interacted with mir-124 to modulate bladder cancer growth, invasion and migration by targeting androgen receptor (ar). Cell Physiol Biochem 2017;43:405-418.

15 Papagiannakopoulos T, Shapiro A, Kosik KS: Microrna-21 targets a network of key tumor-suppressive pathways in glioblastoma cells. Cancer Res 2008;68:8164-8172.

16 Cai J, Guan H, Fang L, Yang Y, Zhu X, Yuan J, Wu J, Li M: Microrna-374a activates wnt/beta-catenin signaling to promote breast cancer metastasis. J Clin Invest 2013;123:566-579.

17 Xu X, Wang W, Su N, Zhu X, Yao J, Gao W, Hu Z, Sun Y: Mir-374a promotes cell proliferation, migration and invasion by targeting srcin1 in gastric cancer. FEBS Lett 2015;589:407-413.

-18 Li J, Ying J, Fan Y, Wu L, Ying Y, Chan AT, Srivastava G, Tao Q: Wnt5a antagonizes wnt/beta-catenin signaling and is frequently silenced by promoter cpg methylation in esophageal squamous cell carcinoma. Cancer Biol Ther 2010;10:617-624.

19 Park HW, Kim YC, Yu B, Moroishi T, Mo JS, Plouffe SW, Meng Z, Lin KC, Yu FX, Alexander CM, Wang CY, Guan KL: Alternative wnt signaling activates yap/taz. Cell 2015;162:780-794.

20 Malgor R, Crouser S, Greco D, Brockett C, Coschigano K, Nakazawa M, Jenkinson S: Correlation of wnt5a expression with histopathological grade/stage in urothelial carcinoma of the bladder. Diagn Pathol 2013;8:139.

21 Seront E, Pinto A, Bouzin C, Bertrand L, Machiels JP, Feron O: Pten deficiency is associated with reduced sensitivity to mtor inhibitor in human bladder cancer through the unhampered feedback loop driving pi3k/akt activation. Br J Cancer 2013;109:1586-1592.

-22 Jiang H, Qu L, Wang Y, Cong J, Wang W, Yang X: Mir-99a promotes proliferation targeting fgfr3 in human epithelial ovarian cancer cells. Biomed Pharmacother 2014;68:163-169. 


\section{Cellular Physiology Cell Physiol Biochem 2018;48:815-826 and Biochemistry Published on/200491911 $2018 \quad \begin{aligned} & \text { DO 2018 The Author(s). Published by S. Karger AG, Basel } \\ & \text { www.karger.com/cpb }\end{aligned}$ \\ Chen et al.: MiR-374a Inhibits Bladder Carcinoma}

23 Goswami CP, Nakshatri H: Progmir: A tool for identifying prognostic mirna biomarkers in multiple cancers using publicly available data. J Clin Bioinforma 2012;2:23.

-24 Nemeth MJ, Topol L, Anderson SM, Yang Y, Bodine DM: Wnt5a inhibits canonical wnt signaling in hematopoietic stem cells and enhances repopulation. Proc Natl Acad Sci U S A 2007;104:15436-15441.

-25 Torii K, Nishizawa K, Kawasaki A, Yamashita Y, Katada M, Ito M, Nishimoto I, Terashita K, Aiso S, Matsuoka M: Anti-apoptotic action of wnt5a in dermal fibroblasts is mediated by the pka signaling pathways. Cell Signal 2008;20:1256-1266.

-26 Fang X, Yu SX, Lu Y, Bast RC, Jr., Woodgett JR, Mills GB: Phosphorylation and inactivation of glycogen synthase kinase 3 by protein kinase a. Proc Natl Acad Sci U S A 2000;97:11960-11965.

-27 Kawasaki A, Torii K, Yamashita Y, Nishizawa K, Kanekura K, Katada M, Ito M, Nishimoto I, Terashita K, Aiso S, Matsuoka M: Wnt5a promotes adhesion of human dermal fibroblasts by triggering a phosphatidylinositol-3 kinase/akt signal. Cell Signal 2007;19:2498-2506. Kypta RM, Waxman J: Wnt/beta-catenin signalling in prostate cancer. Nat Rev Urol 2012;9:418-428.

29 Zhan T, Rindtorff N, Boutros M: Wnt signaling in cancer. Oncogene 2017;36:1461-1473.

-30 Yoshino H, Seki N, Itesako T, Chiyomaru T, Nakagawa M, Enokida H: Aberrant expression of micrornas in bladder cancer. Nat Rev Urol 2013;10:396-404.

31 Topol L, Jiang X, Choi H, Garrett-Beal L, Carolan PJ, Yang Y: Wnt-5a inhibits the canonical wnt pathway by promoting gsk-3-independent beta-catenin degradation. J Cell Biol 2003;162:899-908.

-32 Gujral TS, Chan M, Peshkin L, Sorger PK, Kirschner MW, MacBeath G: A noncanonical frizzled2 pathway regulates epithelial-mesenchymal transition and metastasis. Cell 2014;159:844-856.

-33 Mikels AJ, Nusse R: Purified wnt5a protein activates or inhibits beta-catenin-tcf signaling depending on receptor context. PLoS Biol 2006;4:e115.

-34 Sin MLY, Mach KE, Sinha R, Wu F, Trivedi DR, Altobelli E, Jensen KC, Sahoo D, Lu Y, Liao JC: Deep sequencing of urinary rnas for bladder cancer molecular diagnostics. Clin Cancer Res 2017;23:3700-3710. 